\title{
Aplicación del ciclo de vida y el análisis estructurado en el desarrollo de un laboratorio virtual de transformadores monofásicos
}

\author{
John Milton Ramírez-Romero \& Sergio Rivera-Rodríguez \\ Universidad Nacional de Colombia, Bogotá,Colombia.jmramirezro@unal.edu.co,srriverar@unal.edu.co
}

\begin{abstract}
Resumen - El desarrollo de Laboratorios Virtuales es un campo especial dentro del desarrollo de software debido a las relaciones interdisciplinares que surgen y las características de proyectos de esa naturaleza. En el primer semestre de 2016 se desarrolló, en la Universidad Nacional de Colombia, un Trabajo de Grado titulado Desarrollo de un laboratorio virtual computarizado de un transformador monofásico para el laboratorio de conversión electromagnética dentro del proyecto de investigación formulado: estudio del impacto de la estrategia pedagógica llamada "virtual labs" en el desarrollo de los cursos de laboratorio de máquinas eléctricas e implementación de laboratorio virtual computarizado de transformadores de potencia para la Universidad Nacional de Colombia sede Bogotá. Para hacer realidad el desarrollo del laboratorio virtual, fue necesario integrar temáticas de Pedagogía, Ingeniería de Sistemas e Ingeniería Eléctrica. En este artículo se busca que los lectores que estén interesados en el desarrollo de un Laboratorio Virtual conozcan las directrices básicas necesarias para realizar las etapas iniciales: la fase de análisis y la fase de diseño. Estas fases son críticas ya que describen el sistema actual y definen las características del Laboratorio Virtual, es tal la importancia de estas fases que, de hacerse de forma errónea, conducirá al fracaso del proyecto.
\end{abstract}

Palabras Clave - Análisis de Sistemas, Diseño de Sistemas, Laboratorio Virtual.

Recibido: 24 de agosto de 2016. Revisado: 20 de septiembre de 2016. Aceptado: 28 de septiembre de 2015.

\section{Application of life cycle and structured analysis in the development of a virtual laboratory single phase transformers}

\begin{abstract}
Development of Virtual Labs is a special field within the software development due to interdisciplinary relationships arising and characteristics of projects of that nature. In the first half of 2016 was developed, at the National University of Colombia, a thesis entitled Development of a computerized virtual laboratory of transformers for the electromagnetic conversion laboratory within the research project formulated: Study of the impact of pedagogical strategy called "virtual laboratories" in the development of electric machines laboratory course and implementation of a computerized virtual laboratory for transformers at the National University of Colombia in Bogotá. To realize the development of the virtual laboratory was necessary integrate different subjects as education, systems engineering and electrical engineering. In this paper, readers who are interested in the development of a Virtual Lab know the basic guidelines necessary to perform the initial stages: the analysis phase and design phase. These are critical phases as they describe actual system and define virtual lab features, such it is the importance of these phases, to become so erroneous, will lead to project failure.
\end{abstract}

Keywords — Systems Analysis, Systems Design, Virtual Laboratory.

\section{Introducción}

Para el desarrollo de cualquier proyecto de programación de software es necesario contar con directrices que dirijan el trabajo de forma apropiada, por ello se establecen metodologías que permiten obtener información del sistema actual, se determinen las necesidades a satisfacer con el desarrollo del proyecto y se pueda monitorear su avance.

Si bien se espera que los lectores estén buscando directrices para el desarrollo de sus propios proyectos de Laboratorios Virtuales (de ahora en adelante llamados LV), este documento puede ser consultado por analistas y diseñadores de sistemas y todos aquellos que estén trabajando en proyectos de desarrollo de material pedagógico virtual.

De acuerdo con [1], un LV genera, a través de un ordenador, la combinación de diversas tecnologías para permitir la interactividad en tiempo real de los usuarios con un entorno. En este artículo se muestra como ejemplo el desarrollo del proyecto denominado Laboratorio Virtual de Transformadores Monofásicos (de ahora en adelante llamado LVTM).

Los laboratorios virtuales son una innovación pedagógica en la rama de ingeniería consistente en aplicaciones computarizadas que han revolucionado los ambientes de aprendizaje al poder simular el comportamiento físico de configuraciones experimentales [2]. A través de un computador, un alumno puede recrear las condiciones de una práctica o un experimento contenido en el laboratorio. Esta característica es ideal para recrear el funcionamiento de un sistema cuyo ambiente es potencialmente peligroso antes de que el alumno se enfrente físicamente con las máquinas y equipos reales.

\section{El proyecto LVTM}

Las máquinas con las que cuenta el Laboratorio de Conversión Electromagnética y el equipo para su alimentación, medición y control son costosas. Por lo tanto, no es recomendable que los alumnos operen las máquinas disponibles en condiciones peligrosas $y$ sin un previo entrenamiento.

Estas máquinas eléctricas manejan niveles de tensión y de corriente que pueden causar lesiones serias. De acuerdo al Reglamento Técnico de Instalaciones Eléctricas [3], existen

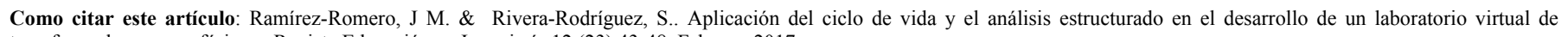
transformadores monofásicos . Revista Educación en Ingeniería 12 (23) 43-48, Febrero, 2017. 
estudios que determinan la soportabilidad de los seres humanos y animales que relacionan la corriente eléctrica, el tiempo de exposición a ella y el riesgo fisiológico (asociado a la ocurrencia de fibrilación, rigidez muscular, amputación de algún miembro del cuerpo y la muerte).

Una buena parte de los riesgos mencionados se pueden reducir si los alumnos conocen de antemano qué puede salir mal. Los Laboratorio Virtuales se presentan con una estrategia innovadora pedagógica para hacer frente a las desventajas mencionadas, ya que realizarán la misma practica q harán en el laboratorio, pero en un ambiente de aprendizaje virtual, y en este ambiente se darán cuenta de los efectos de realizar malas conexiones o condiciones no solo para los equipos sino también los efectos en las personas [4,5].

El proyecto LVTM consistió en el desarrollo de un software que permite recrear las mediciones efectuadas en transformadores monofásicos durante los ensayos de medición de resistencia de devanados, de vacío, de corto circuito, regulación y eficiencia. Fue desarrollado como una aplicación bajo plataforma Windows (ver Fig. 1). En los laboratorios reales, estas actividades se desarrollan empleando un transformador monofásico real, instrumentos de medición (como multímetros y analizadores de redes) y una instalación eléctrica dotada con elementos de alimentación y protección.

Los niveles de corriente eléctrica empleados ponen en riesgo la integridad física de las personas, el equipo y la infraestructura del laboratorio. De acuerdo al Reglamento Técnico de Instalaciones Eléctricas, los niveles de corriente empleados en los ensayos descritos anteriormente pueden producir quemaduras de segundo y tercer grado, tetanización y daños cardiacos. Hay que tener en cuenta que los tiempos de recuperación son extensos y que el dolor físico y emocional que los acompaña es muy elevado.

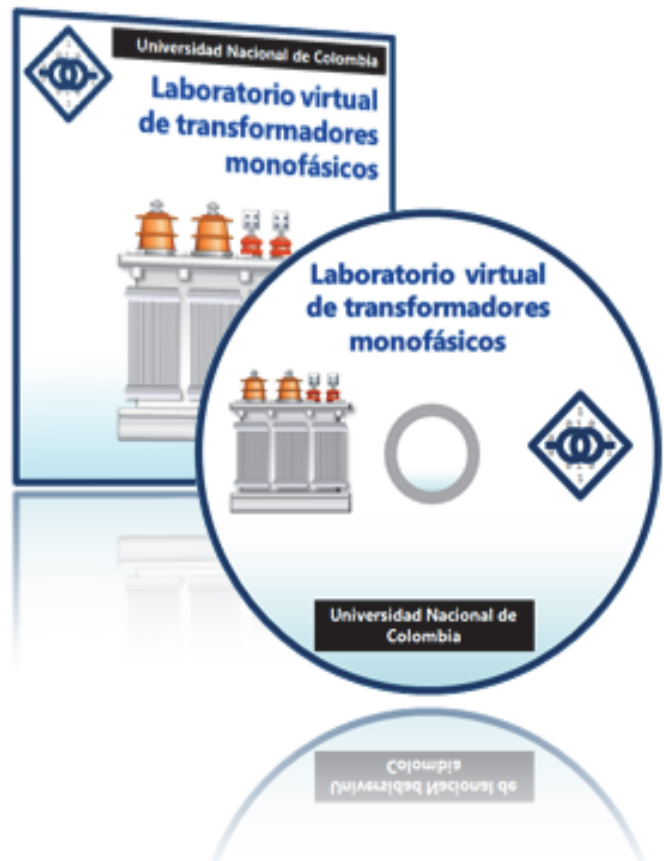

Figura 1. DVD del Laboratorio Virtual de Transformadores Monofásicos. Fuente: Los autores
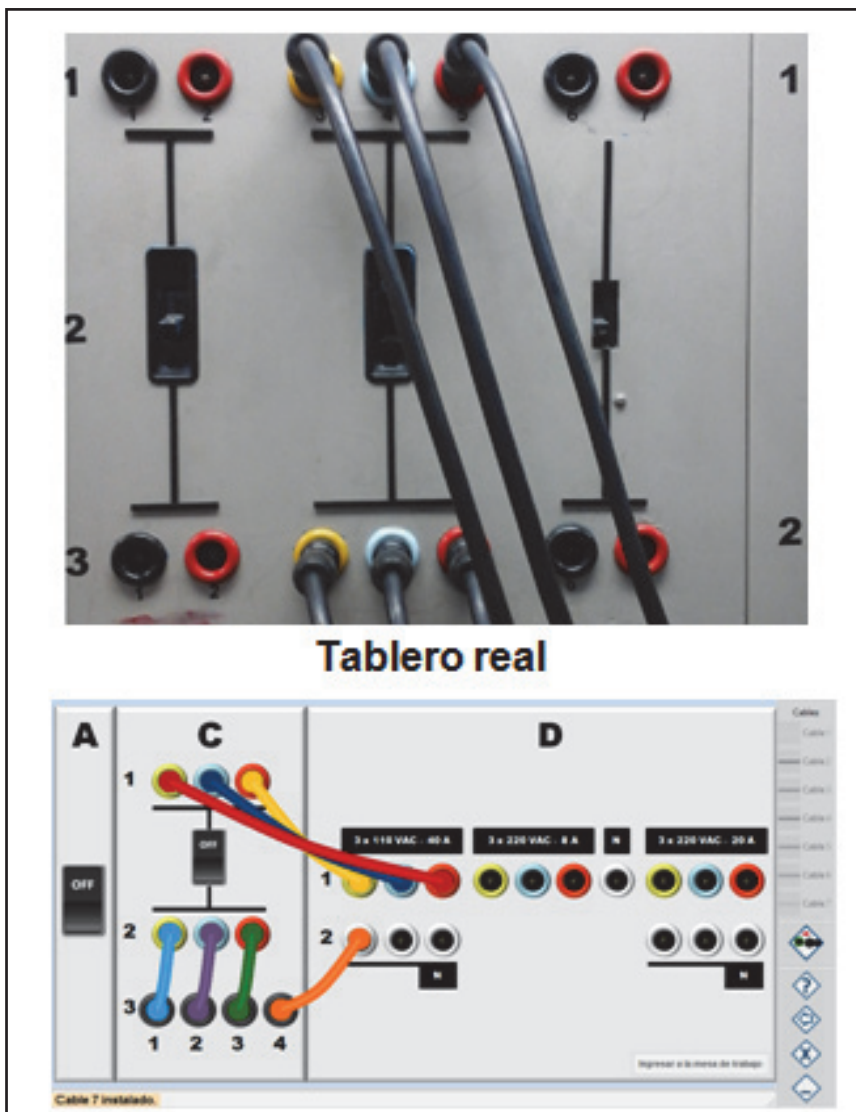

Tablero del Laboratorio Virtual

Figura 2. Comparación entre una sección de un tablero de protecciones real y su representación en el Laboratorio Virtual.

Fuente: "Los autores

Dentro del diseño de las pantallas del LVTM se tuvo cuidado de representar los elementos usados en situaciones reales lo más cerca posible de la realidad, algunos elementos pedagógicos se tomaron en cuenta (como, por ejemplo, el uso de colores en los cables para facilitar la visualización de las conexiones). Puede verse el comparativo en la Fig. 2.

A diferencia de los desarrollos de software que suelen realizarse dentro del entorno académico (como simulaciones de campos electromagnéticos mediante software que emplea estados finitos), los LV son proyectos de un tamaño mayor, esto se debe a la cantidad de disciplinas involucradas en el proceso $[6,7]$.

En la parte superior de la Fig. 3 se muestra un montaje de laboratorio empleado para el ensayo de circuito abierto a un transformador monofásico. Los gráficos de los cables del LVTM (parte inferior) pueden parecer desordenados, pero estas conexiones son las que se encuentran en la vida real (se debe aclarar que la representación más ordenada conllevaría al empleo de líneas rectas, pero no se puede lograr con cables reales). También se puede cambiar la proporción de las dimensiones de los instrumentos para hacer énfasis a los usuarios en lo que se estima que es importante (nótese que la pantalla azul, la cual es un Analizador de Redes, es mucho más grande en el LVTM que en el Laboratorio real). 


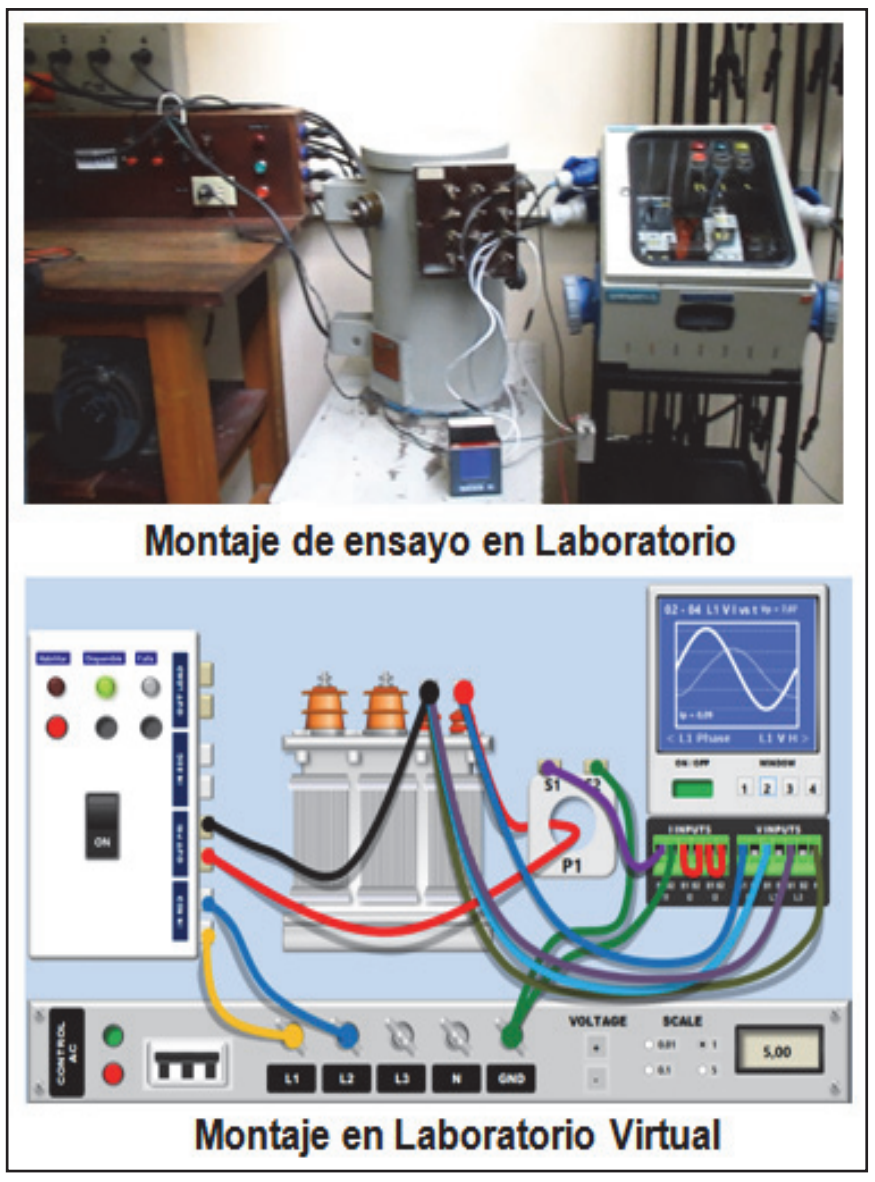

Figura 3. Comparación entre una sección de un tablero de protecciones real y su representación en el Laboratorio Virtual.

Fuente: Los autores

\section{Descripción de proyectos de LV usando diagramas de Venn}

Como se asume que en los proyectos de LV siempre existirán relaciones interdisciplinarias, estas se describen de una forma apropiada si se usa un diagrama de Venn. Los lectores pueden entender fácilmente la relación entre ellas, y aunque las disciplinas involucradas pueden ser diferentes el producto final es el mismo: un LV.

La Fig. 4 describe las relaciones entre las disciplinas involucradas en el proyecto LVTM. El desarrollo de un software requiere del estudio de las actividades actuales del laboratorio, por lo que se requiere seguir directrices para ese fin, las usadas en el proyecto LVTM son el Ciclo de Vida de Sistemas y el Análisis Estructurado (C.V.S.-A.E.), las cuales son usadas en la Ingeniería de Sistemas desde décadas atrás en el análisis de sistemas corporativos (como los encontrados en los bancos). Así mismo, los modelos matemáticos de circuitos eléctricos permiten describir el comportamiento del transformador y los dispositivos de medición, son empleadas en Ingeniería Eléctrica desde inicios del siglo XX. Como el software se elabora con fines educativos, es necesario recurrir a la pedagogía para establecer directrices de diseño.

Nótese que el diagrama de Venn mostrado en la Fig. 4 tiene intersecciones entre las disciplinas involucradas: la intersección entre el C.V.S-A.E. con la pedagogía representa las directrices de diseño de software educativo, cuando estas se cumplen se obtiene un Objeto Virtual de Aprendizaje (O.V.A.). La intersección entre el C.V.S-A.E. y los modelos matemáticos de transformadores representa las aplicaciones hechas en computador que permiten agilizar y/o automatizar cálculos, a estas se le denominan Algoritmos (tal vez el cálculo de tensiones de entrada y de salida de un transformador sólo requiera la solución de un cociente, pero cuando se necesita recrear la medición de la resistencia de sus devanados se deben involucrar algoritmos gráficos, cálculos matemáticos y máquinas de estado finito simultáneamente).

Cuando en el entorno pedagógico se requiere representar el funcionamiento de un transformador a un auditorio académico es muy probable que se quiera ir más allá de las cifras y las fórmulas y se quiera mostrar un comportamiento en tiempo real y de forma gráfica (por ejemplo, un corto circuito), en ese caso se estará hablando de una Simulación. Cumpliendo con los parámetros de diseño de un O.V.A. y elaborando algoritmos que permitan el funcionamiento de un conjunto de simuladores, se obtiene un LV.

\section{Análisis de sistemas en proyectos de desarrollo de LV}

James Senn, autor del libro Análisis y Diseño de Sistemas de Información [8] describe el análisis y diseño de sistemas como el examen de la situación actual de una o más áreas de una institución para determinar qué aspectos pueden mejorarse mediante la aplicación de métodos y procedimientos más adecuados que los actuales. El analista de sistemas debe enfocar su atención en el funcionamiento actual del sistema, determinar el origen de la información, su empleo y los motivos por los cuales se deben hacer cambios. De esa manera, podrá indicar los beneficios y los perjuicios que se pueden derivar con el desarrollo e implementación de un Laboratorio Virtual. El análisis de sistemas puede ir más allá de una descripción: también puede ser usado para indicar cómo se puede dirigir la expansión de una corporación.

En el proyecto LVTM, el análisis se centró en las actividades realizadas con los transformadores monofásicos. Durante el pregrado los alumnos deben ver y aprobar la materia Conversión Electromagnética, una de las primeras unidades que se estudian se enfoca en los modelos matemáticos del transformador monofásico. Posteriormente, se emplean modelos similares para describir a los transformadores trifásicos y algunas otras máquinas eléctricas. En esta etapa los alumnos nunca tienen contacto con las máquinas eléctricas involucradas.

Por otro lado, la Universidad Nacional ofrece a los alumnos la materia electiva Laboratorio de Conversión Electromagnética, esta materia se enfoca en la demostración práctica de los principios vistos en Conversión Electromagnética. Allí es donde se detectó una de las necesidades que puede satisfacer el desarrollo del LVTM: puede que todos los estudiantes estén capacitados en el uso de los modelos matemáticos de un transformador monofásico, pero muy pocos son los que llegan a tener la oportunidad de operarlos. Con el desarrollo del LVTM, los docentes y los alumnos pueden comprender de dónde se obtienen los datos con los que se realizan los modelos equivalentes realizando una simulación de los ensayos efectuados en un laboratorio sin el riesgo de sufrir un accidente. 


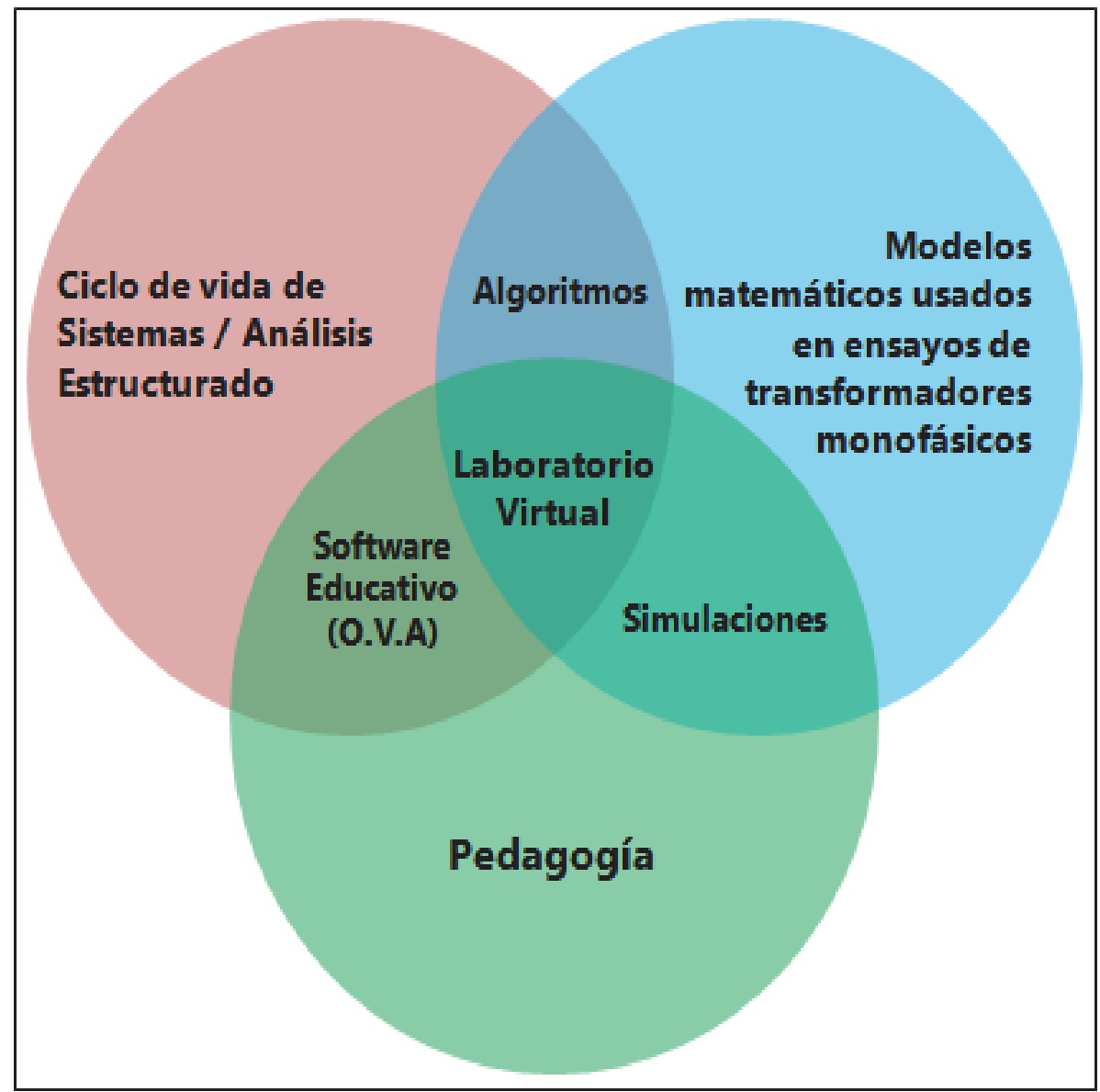

Figura 4. Disciplinas involucradas en el proyecto LVTM.

Fuente: Los autores.

Para la tarea de recolección de datos y descripción del sistema actual, la literatura sugiere el empleo de entrevistas y recolección de datos (sobre todo, los manuales que describen los procesos). El factor crítico en esta fase es la experiencia, en la medida de lo posible es necesario que dentro del proyecto se involucre al personal que realice los montajes de los experimentos en el entorno real. Actualmente es muy sencillo grabar videos, un solo archivo de vídeo puede ser más útil que un manual técnico completo. La observación es muy importante para seguir detectando los beneficios del desarrollo de un LV, durante la fase de análisis del proyecto LVTM se encontró que los estudiantes que apenas empiezan a manejar el transformador real muestran un temor irracional al momento de realizar las conexiones (aunque tengan los planos de conexiones a la mano), sólo puede ser superado por los alumnos que han realizado exitosamente esta tarea. Se espera que al implantar el LVTM en el Laboratorio de Máquinas Eléctricas, los alumnos manejen el LVTM y puedan superar el temor inicial más rápido [9].

\section{Diseño de sistemas en proyectos de desarrollo de LV}

Una vez que se conoce el sistema actual y los cambios que pueden realizarse, se debe establecer una metodología para que el LV sea capaz de satisfacer las necesidades detectadas en la Fase de Análisis. Como es de suponerse, no todas las necesidades se pueden satisfacer y es un error pensar que un LV deba ser diseñado para que describa fielmente la realidad, sólo es necesario que describa el funcionamiento dentro de condiciones controladas.

En el desarrollo del LVTM, se tuvo en cuenta que los ensayos se realizan con instrumentos y conexiones diferentes entre sí, por lo tanto, se decidió hacer una pantalla por cada ensayo. Los errores más frecuentes (y que son más costosos) se deben a la conexión errónea de multímetros en modo de medición amperimétrica con fuentes de tensión (ya que su resistencia es muy pequeña, la corriente presente entre las terminales del multímetro puede exceder su corriente límite, por lo que se quema su fusible). En otros casos, como en el ensayo de vacío y de corto circuito, se optó por impedir que el usuario sea capaz de llevar la fuente de alimentación más allá de la tensión y la corriente nominal del transformador (datos obtenidos a valores más alto no tienen sentido cuando lo único que se quiere es obtener los datos para calcular los parámetros de un circuito equivalente).

En general, para el desarrollo de cualquier LV, se aconseja seguir las directrices descritas por Senn (ver Fig. 5):

- Iniciar con el diseño de las salidas, en el caso de los LV la mayor parte de la atención se centra en la pantalla, por lo que es necesario examinar el aspecto de los elementos involucrados y dibujarlos de tal manera que se parezcan a los reales. 


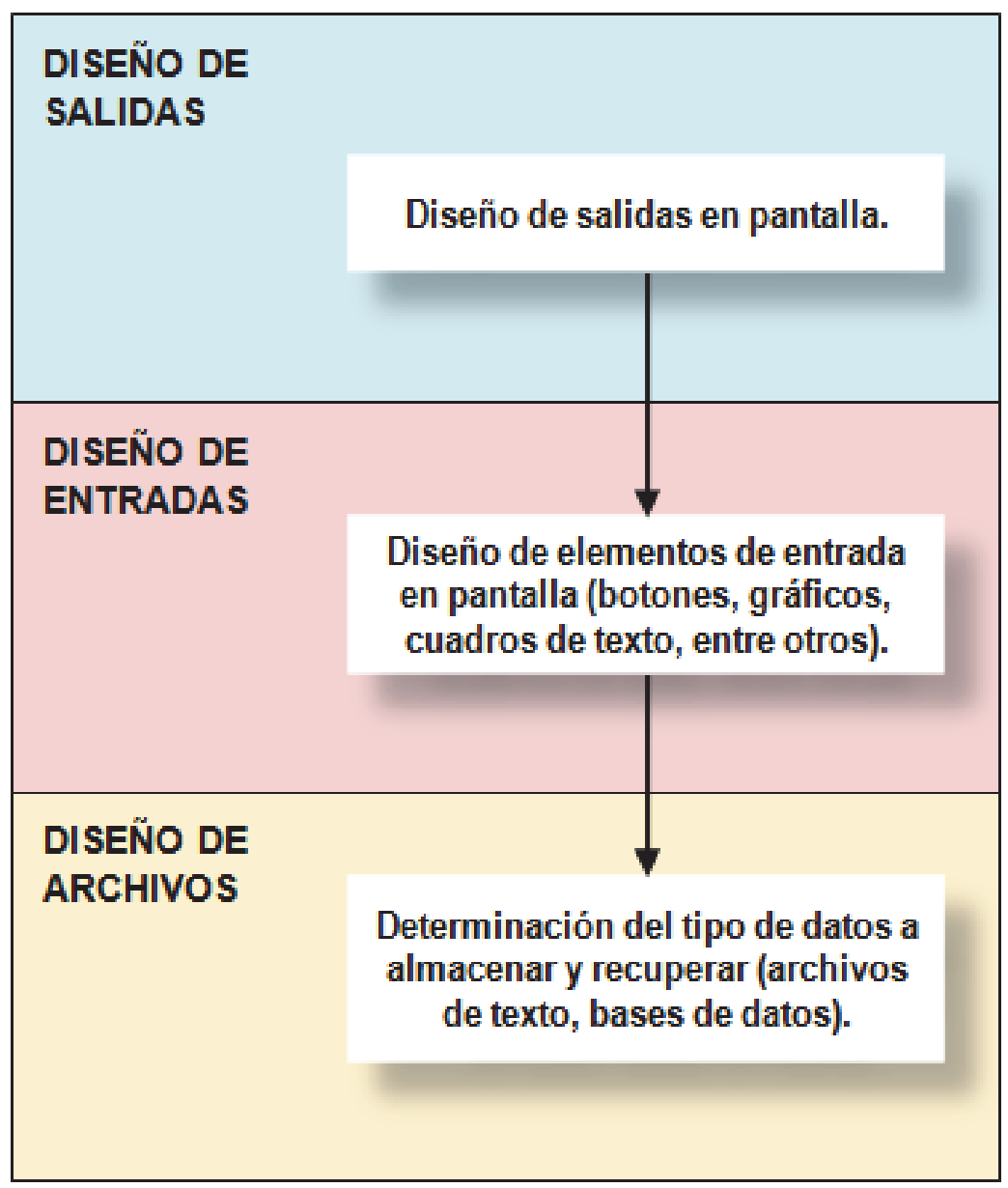

Figura 5. Directrices para el desarrollo de la fase de diseño en un Laboratorio Virtual.

Fuente: Los autores.

- El diseño de las entradas en un LV se centra en la forma en la que el usuario ingresa los datos (como, por ejemplo, dónde van conectados los cables y las escalas del multímetro). Aquí es necesario utilizar un modelo de caja negra donde el software detecta las entradas (por ejemplo, la posición del mouse al hacer clic), y con base a ellas defina qué debe hacer (un ejemplo es la conexión de un cable a las terminales de una fuente de tensión encendida, si eso sucede entonces ocurrirá un corto circuito). Aquí es muy útil emplear máquinas de estado finito, para los lectores que no están familiarizados con este término se puede decir que es un software o un hardware que hace sólo una actividad y emite una salida dada sólo si hay un determinado tipo de entrada.

- La siguiente etapa es el diseño de archivos, aquí se define qué es lo que debe almacenar y recuperar el LV. La estrategia más sencilla es emplear archivos de texto, para desarrollos más complejos se aconseja el uso de bases de datos. Es muy importante tener en claro el tipo de datos que se va a almacenar y el tipo de datos que se usará (un error muy frecuente es el almacenamiento directo de variables tipo boolean, por lo que es necesario conocer plenamente como se usa dentro de la plataforma de desarrollo).

\section{Conclusiones}

El desarrollo de un LV presenta variantes con respecto al desarrollo de software corporativo (en donde el desarrollo se centra en el manejo de información en bases de datos). En un $\mathrm{LV}$, el factor determinante es el manejo de información de posiciones, conexiones y salidas gráficas.

Los proyectos de desarrollo de LV, independientemente del campo al cual será dirigido, siempre tendrá relaciones interdisciplinarias.

El desarrollo apropiado de las fases de análisis y diseño es fundamental para el éxito de un proyecto de desarrollo de un LV. Las habilidades de los programadores y de los dibujantes también deben de tenerse en cuenta para fijar los límites que tendrá el LV. Las necesidades de los usuarios dependen del entorno donde se operará el LV.

Ya que la información necesaria para el desarrollo apropiado de la fase de análisis y de diseño de cualquier proyecto de software es extensa, conviene tomar las variantes presentadas en este artículo para el desarrollo de cualquier LV. Por ejemplo, el uso del diagrama de Venn puede parecer trivial, pero sin él puede que no se tenga claro cuál es la contribución 
de cada una de las disciplinas involucradas en los proyectos de desarrollo de LV.

\section{Referencias}

[1] Farrerons, O. y Olmedo, N., Las TIC y la ingeniería gráfica, Barcelona: Omnia Science Monographs, 2016. DOI: 10.3926/oms.306.

[2] iitg.vlab.co.in, Virtual Lab: Magnetic Field Behaviour in single coil, 16 March 2011. [Online]. Available: iitg.vlab.co.in/?sub=61\&brch=168\&sim=1050\&cnt=1918. [Accessed 20 August 2016].

[3] Ministerio de Minas y Energía, Reglamento Técnico de Instalaciones Eléctricas RETIE, Bogotá D.C.: República de Colombia - Ministerio de Minas y Energía, 2005.

[4] Milano, F., Vanfretti, L. and Morataya, J., An open source power system virtual laboratory: The PSAT case and experience, IEEE Transactions on Education, 51(1), pp. 17-23, February 2008. DOI: 10.1109/TE.2007.893354

[5] Benetazzo, L., Bertocco, M., Ferraris, F., Ferrero, A. and Offell, C., A Web-based distributed virtual educational laboratory, IEEE Transactions on Instrumentation and Measurement, 49(2), pp. 349-356, April 2000. DOI: 10.1.1.467.6756

[6] Achumba, I., Azzi, D., Dunn, V. and Chukwudebe, G., Intelligent performance assessment of students' laboratory work in a virtual electronic laboratory environment, IEEE Transactions on Learning Technologies, 6(2), pp. 103-116, 2013. DOI: 10.1109/TLT.2013.1

[7] Castillo, A., Ortegon, J., Vazquez J. and Rivera, J., Virtual laboratory for digital image processing, IEEE Latin America Transactions, 12(6), pp. 1176 - 1181, 2014. DOI: 10.1109/TLA.2014.6894017

[8] Senn, J., Análisis y diseño de sistemas de información, México D.F.: Mc. Graw Hill, 1998.

[9] Ramirez, J., Desarrollo de un laboratorio virtual computarizado de un transformador monofásico para el laboratorio de conversión electromagnética, Trabajo de grado, Universidad Nacional de Colombia, Bogotá, Colombia, 2016.

J.M. Ramírez-Romero, es Ing. Electricista de Universidad Nacional de Colombia (2016), Bachiller Técnico en Sistemas y Computación del Instituto Técnico Industrial Piloto; programador aficionado desde 1998

ORCID: 0000-0002-0982-2284

S. Rivera -Rodríguez, es PhD. Ing. Electricista de Universidad Nacional de Colombia (2001); Esp. en Ingeniería Eléctrica con énfasis en Sistemas de Distribución, $\mathrm{PhD}$ en Ingeniería Eléctrica del Instituto de Energía Eléctrica, Universidad Nacional de San Juan (2011). PhD. asociado en el MIT Massachusetts Institute of Technology (2013); profesor en Universidad Nacional de Colombia en el área de sistemas de potencia y máquinas eléctricas (2014).

ORCID: 0000-0002-2995-1147 